\title{
Interactive comment on "Global analysis for periodic variations of gravity wave squared amplitudes and momentum fluxes in the middle atmosphere" by Dan Chen et al.
}

\section{Dan Chen et al.}

nuistcd@163.com

Received and published: 16 May 2019

Dear Dr Pisoft,

We would like to sincerely thank both reviewers for their positive and helpful comments. Please find below a point to point reply. We have all points taken into account in the new revised version of the paper.

Thank you very much for handling this manuscript.

Dan Chen 
General comments: This paper is very well written, the analyses methods are up to date and have been carried out with the utmost care. Only two aspects on why data are treated a special way should be clarified. Even though it would be nice to have a global GW momentum flux including propagation direction, the analysis method used here is the best we have at the moment as far as I know. The authors give also a very good and extensive discussion missing only a few points (see below) and summarize their results in clear schemes. Therefore I strongly recommend to publish this study after a minor revision.

Reply: We thank the reviewer for the very positive recommendation and the detailed comments. We have taken all comments into account in the revised manuscript and give respective replies (in blue) below.

Specific comments: 1) SABER data are available from January 2002 until today. Why do the authors use data only between 2002 and 2015 and do not include at least also 2016 and $2017 ?$

In our publication we use the referenced data set of the GRACILE climatology which is documented in Ern et al., ESSD, 2018 and which is publicly available under https://doi.org/10.1594/PANGAEA.879658, While SABER retrievals are continuously performed and released, the GW analysis is not a standard product and we here preferred to base on the published climatology. We have included a sentence in the revised manuscript: 'This reference data set comprises the years 2002-2015 and we base our analysis on this period.' on page 5, line 13.

2) Monthly averaged GWSTA and GWMF have a grid resolution of $10^{\circ}$ in longitude and $5^{\circ}$ in latitude. To obtain values at each grid point the data are averaged in grid boxes of $30^{\circ}$ in longitude and $20^{\circ}$ in latitude. This means that the averaged grid boxes are three or four times larger than in the initial calculation. Is this really necessary, especially in the latitude direction?

This again is due to our decision to use the GRACILE data set. For GRACILE the zonal 
means are calculated from the global maps. This has the advantage of a higher selfconsistency of the data set. Furthermore, it harmonizes differences in the sampling. We have added an according sentence in the revised manuscript: 'In order to avoid biases through inhomogeneous sampling we base our zonal means on global maps.' on page 5 , line 8 .

3) Figure 1a shows the FFT spectrum of the GWMF at $45^{\circ} \mathrm{N}$. Besides the peaks discussed here (AV, SAV, TAV and QBO) there is also a peak around 6-7 years which is even higher than that one of QBO. I assume that this peak results from ENSO (EI Nino southern oscillation). Please shortly discuss this peak even though you will not go into much detail in your further analyses.

In Fig. 1a there is indeed a peak at around 6-7 years and also a peak at 13 years higher than QBO. From the horizontal axes in Fig.1a, it can be seen that the high resolution of periods analyzed by FFT concentrates below 3 years, while low resolution exists in 3-13 years. In addition, Fig. 1b shows the significant periods: in particular these are found for values below 3 years over a wide range of latitudes. For this reason, we only focus on the annual, semiannual, terannual, quasi-biennial periods as dominating oscillations.

Based on your suggestions, we have added a short discussion about this peak in our revised paper: 'In addition to the above-mentioned four major frequencies there are two peaks at around 6-7 years and 13 years. Due to the length of the time series of 13 years (156 months dataset), these are the two lowest frequencies (longest periods) which potentially could be resolved by FFT. These lowest frequencies are more likely to be influenced by leakage and aliasing. Therefore, we focus on the periods of 2.167, $1.0,0.5$ and 0.333 years, which are contained for several cycles and hence well constrained by our data. Potential explanations for the longer periods are ENSO which is an interannual oscillation of 2-7 years and the 11-year solar cycle.' on page 7, line29.

4) Even though the Southern Hemisphere experienced only one SSW in the last 
decades namely in 2002, does make it a difference for the spectral analysis including or excluding this year? I am convinced of your results but I am also curious about the difference.

Basing on your comment we have repeated the FFT excluding the year 2002 . The resulting variance difference between excluding and including this year is shown in Fig. $1 \mathrm{R}$ of this reply. Figure $1 \mathrm{Rc}$ and $1 \mathrm{Rd}$ are the same as Fig. 9e and $9 \mathrm{f}$ in our manuscript and display the fraction of variance explained by the four main periods. The upper row shows the results excluding 2002, the middle row the results including 2002 and the lower row the difference. Differences in the explained variance are generally small. In the latitude range of the southern hemisphere vortex there is a general increase of about $3 \%$ by excluding 2002 . The largest difference is found in regions with a strong QBO signal, which is difficult to describe by a single, fixed period (cf. discussion in text) and which is better described when shortening the data set. We have inserted a sentence in the revised manuscript: 'The exception from this is the split-event of the southern polar vortex in 2002. We have tested our analysis (not shown) and find only a very minor impact on our results. Different from this continuous descent of the phase of the southern polar vortex, the breakdown ... on page 9, line 30.

5) The described variance by the four variations in mid-latitude upper stratosphere is higher in the southern hemisphere than in the northern hemisphere. An additional cause for this effect might be a general much higher Rossby wave activity in the northern than in the southern hemisphere resulting in a much more variable polar vortex in the northern hemisphere and therefore in a much more variable GW filtering.

Based on your suggestion and also based on comment 2 of the second reviewer we have added a short discussion about this effect in our revised paper: '.. is higher in the southern than in the northern hemisphere. Several effects may play a role. First, planetary wave activity is much higher in the northern than in the southern hemisphere. This results in higher variability in GW filtering. Likely more important for our time series of monthly zonal means is that this higher PW activity also frequently induces sudden

Interactive

comment 
stratospheric warmings, which terminate the ... on page 15, line 22.

6) Figure 10 shows the GW variation related to the 11-year solar cycle. The authors ANGEOD calculated the median value of the time series and defined the beginning and ending of this solar cycle by the occurrence of "valleys". However, the dependency of the GW variation on the solar cycle is more pronounced in the GWMF than in the GWSTA since there is a third "valley" around 2009 even though it was still above the median. The solar minimum in 2009 was the lowest solar minimum of the last decades. Please discuss this the different behavior between GWMF and GWSTA around this solar minimum.

Since the coupling of the solar cycle is not really understood, it is difficult to speculate about any potential reasons. We have included a sentence to emphasize the difference between GWMF and GWSTA: 'This is more pronounced in absolute GWMF than GWSTA. The latter even has a small local minimum around 2009 which is close to the time of the solar minimum. The stronger solar cycle in absolute GWMF means that in particular GWs of short horizontal and long vertical wavelengths are modulated, which are more directly coupled to convective sources.' on page 16, line 2. And, in addition: '. . . convective activity consistent with the stronger modulation of absolute GWMF. The fact that we find some indications of absolute GWMF variations at timescales of around 11 years in the presence of other, stronger modulations, may be also due to $2008 / 2009$ being the lowest solar minimum of the last decades. For more conclusive analyses several cycles of the investigated period would be needed which is still a long way to go from present day data sets.' on page 16, line 14.

Technical comments: P2L24: . . other sources are accounted for in a so-called...

P3L26: Li et al. (2016) further found an indication ... (tense for consistency)

P11L20: ...structures in Fig. 4a with Fig. 3c especially above 60km and Fig. ... (similar structures are more obvious above 60km)

P12L21: ...four month period (i.e. May/September/January) in the Southern

Interactive

comment
Printer-friendly version

Discussion paper 
Hemisphere... (for consistency with Fig. 5b)

Reply: Implemented as suggested.

ANGEOD

P13L17: ... use the number index 1 to 26 for the colorbar. -> Does month 1 presents January? Please clarify that in the text.

We have clarified this in our revised paper: 'According to the dominant QBO period, we

Interactive

comment use 26 months for the phase display of the QBO. That means the phase shifts roughly one month per calendar year with respect to e.g. January; in other words, the fixed month allocation of phases we can use for the other periods does not work for QBO and the month-index 1-26 indicates a different calendar month in every year.' on page 13, line 33 .

P14L20: One possible reason could be that the study of Krebsbach and Preusse (2017) ...

Figure 1: Example of an FFT ...

Reply: Implemented as suggested.

Please also note the supplement to this comment:

https://www.ann-geophys-discuss.net/angeo-2019-31/angeo-2019-31-AC1-

supplement.zip

Interactive comment on Ann. Geophys. Discuss., https://doi.org/10.5194/angeo-2019-31, 2019. 
ANGEOD
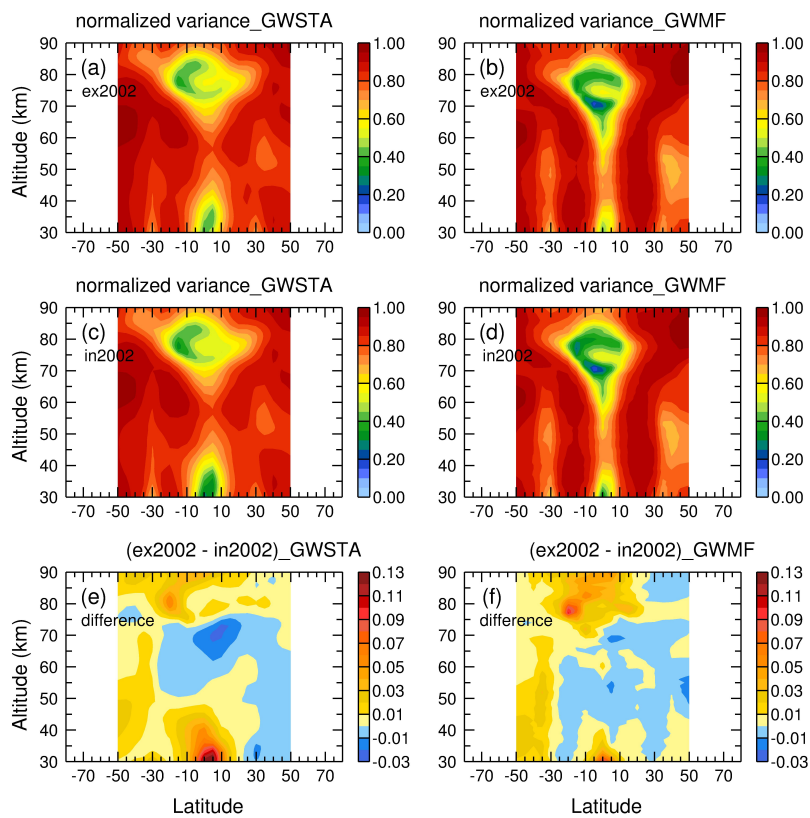

Interactive

comment

Fig. 1. Figure 1R. The variance of GWSTA and absolute GWMF for excluding ( $a, b)$ and including (c, d) the year 2002, and difference (e, f) between them. 